\title{
Tableros de partículas y MDF de Eucalyptus nitens: Ensayos a escala industrial
}

\author{
Particleboard and MDF using Eucalyptus nitens: Industrial scale experiments \\ BRUNO GORRINI, ${ }^{1}$ HERNAN POBLETE, 2 GONZALO HERNANDEZ, ${ }^{3}$ FERNANDO DUNN .4 \\ ${ }^{1}$ Jefe de Investigación y Desarrollo MASISA S.A., E-mail: bgorrini@masisa.cl \\ 2 Inst. Tecnología de Productos Forestales, Universidad Austral de Chile, E-mail: hpoblete@uach.cl \\ 3 Investigador Instituto Forestal de Chile, CORFO, E-mail: ghernand@infor.cl \\ ${ }^{4}$ Subgerente Desarrollo Silvícola MININCO S.A., E-mail: fdunn@formin.cmpc.cl
}

\section{SUMMARY}

Particleboard containing 20\% Eucalyptus nitens, and MDF consisting of 100\% Eucalyptus nitens wood were produced industrially at two plants owned by the company MASISA S.A. Normal production parameters for Pinus radiata were maintained. The particleboard produced had the same mechanical and physical properties that MASISA S.A. achieves under normal conditions for Pinus radiata. The MDF panels containing E. nitens did not reach industrial standards. Further investigations are necessary to achieve the required mechanical and physical property standards for MDF boards. The investigation of the properties of these panels indicated that with some process corrections, it will be possible to produce MDF using Eucalyptus nitens.

Key words: Eucalyptus nitens, particleboard, MDF, medium density fiberboard.

\section{RESUMEN}

En dos plantas industriales de MASISA S.A. se fabricaron tableros de partículas y MDF empleando madera de Eucalyptus nitens. La madera de E. nitens reemplazó a un $20 \%$ del tradicional Pinus radiata en la fabricación de tableros de partículas y en un $100 \%$ en MDF. Los parámetros de producción permanecieron tal como se fijan para la producción con pino radiata. Las propiedades de los tableros de partículas cumplieron con los estándares que se fija MASISA S.A. para su producción normal. Los tableros MDF no lograron satisfacer estos estándares. Se recomienda continuar la investigación para mejorar las propiedades de los tableros MDF. Pese a lo anterior, los valores obtenidos permiten aseverar que, con algunas correcciones al proceso, se podrán producir tableros MDF con Eucalyptus nitens.

Palabras clave: Eucalyptus nitens, tableros de partículas, MDF, tableros de fibras de densidad media.

\section{INTRODUCCION}

Durante los últimos 20 años en Chile se han plantado alrededor de 140.000 ha de Eucalyptus nitens, siendo una especie que ha mostrado tener un muy rápido crecimiento, aproximadamente 30 $\mathrm{m}^{3} / \mathrm{ha} /$ año (1). Esta elevada tasa de crecimiento permite efectuar cosechas tempranas de madera delgada, con edades entre 8 y 12 años. En rodales algo más maduros, con edades a partir de 15 años, se espera poder cosechar madera con diámetros que permitan la producción de madera aserrada y contrachapados.

En determinaciones realizadas en la madera de $E$. nitens cosechada con edades entre 15 y 16 años se ha registrado una densidad básica promedio de $510 \mathrm{~kg} / \mathrm{m}^{3}$ (2).

Considerando que en trozos más jóvenes la densidad debiera ser aún menor y que esos niveles de densidad básica permitirían el uso de esta 
BOSQUE 25(3): 89-97, 2004

Tableros de partículas y MDF de Eucalyptus nitens: Ensayos a escala industrial

especie en la fabricación de tableros reconstituidos, se procedió a hacer dos ensayos a escala industrial, cuyo propósito fue tener una primera visión de las opciones del E. nitens ante este tipo de tecnología. Los tableros a producir fueron de partículas y de fibras de densidad media (MDF).

\section{MATERIAL Y METODOS}

La materia prima empleada en el ensayo, correspondió a Eucalyptus nitens de 5 y 13 años, cosechado en predios de la empresa FORESTAL MININCO S.A. que se ubican en la zona de Mulchén, Octava Región, Chile. Para fabricar los tableros se cosecharon en total $304 \mathrm{~m}^{3}$. En el caso de la elaboración de MDF se ocuparon $112 \mathrm{~m}^{3}$ de 5 años y $96 \mathrm{~m}^{3}$ de 13 años. La inclusión de E. nitens en tableros de partículas consideró 48 $\mathrm{m}^{3}$ de cada edad.

Para la fabricación de los tableros se dispuso de dos plantas industriales de la empresa MASISA S.A. En estas fábricas se procesaron las trozas manteniendo todas las condiciones que normalmente aplican estas plantas para la elaboración de sus tableros con Pinus radiata.

Los ensayos posteriores, determinaciones de propiedades físicas y mecánicas, se efectuaron aplicando las normas europeas EN. Las propiedades determinadas fueron: densidad, hinchamiento, absorción, humedad, emanación de formaldehido, flexión estática (MOR y MOE), tracción perpendicular, desprendimiento superficial y extracción de tornillos (caras y cantos).

Fabricación de tableros de partículas: Los ensayos industriales se realizaron en la planta Chiguayante de MASISA S.A. En esta planta la mezcla de maderas que normalmente se emplea en la fabricación de tableros es de $80 \%$ de aserrín y viruta y de un $20 \%$ de virutas obtenidas de madera en trozos (metro ruma). Toda la madera empleada corresponde a Pinus radiata. Considerando esta composición de la materia prima y que en el caso de E. nitens se trata de trozas obtenidas de un raleo, sólo se reemplazó el 20\% de trozos de $P$. radiata (metro ruma) por trozos de E. nitens.
Con el objeto de poder comparar los resultados de E. nitens con la producción normal de $P$. radiata, las condiciones de fabricación (velocidad de producción, temperaturas, niveles de encolado, presión, entre otros) se mantuvieron constantes e idénticas a las aplicadas cuando se procesa un $100 \%$ de $P$. radiata.

La única variación estudiada en este ensayo fue la edad de la madera, habiéndose utilizado madera de 5 y 13 años para la producción de tableros diferentes. La duración total del ensayo correspondió a 4 turnos de producción, dos para cada edad de la madera.

La madera de $E$. nitens fue procesada con una viruteadora de tambor (Homback) con las condiciones de operación normales. La planta cuenta con una prensa Siempelkamp discontinua de 7 platos. Los tableros producidos eran de tres capas, encolados con Urea formaldehido y de 15 $\mathrm{mm}$ de espesor. La densidad nominal de los tableros se fijó en $640 \mathrm{~kg} / \mathrm{m}^{3}$, el mismo valor que se emplea para el caso de la fabricación con $P$. radiata.

Fabricación de tableros de fibras de densidad media $(M D F)$ : El ensayo de tableros MDF se llevó a cabo en la planta de MASISA S.A. Cabrero. Esta planta posee un desfibrador Sunds (L46) y una prensa continua de $21 \mathrm{~m}$ (Küsters).

En este caso la planta se abastece de astillas de pino radiata. Para el ensayo se astillaron las trozas de E. nitens en la planta de astillado de Soc. Agrícola y Forestal Pozo y Reyes Ltda. en la localidad de Los Angeles, y se fabricaron tableros MDF con un $100 \%$ de esta especie. Lo anterior significó detener la planta, limpiar los conductos y equipos, y continuar la producción con las astillas del eucalipto.

Los tableros fabricados fueron encolados con Urea formaldehido fortificada con Melamina. El espesor nominal se fijó en $15 \mathrm{~mm}$ y la densidad en $600 \mathrm{~kg} / \mathrm{m}^{3}$.

Tal como en el caso de los tableros de partículas, se incluyó como variable la edad de la madera procesada, 5 y 13 años. La duración del ensayo fue de 8 horas de producción.

En los cuadros 1 y 2 se entrega una detallada lista con los parámetros de los procesos en las plantas de Chiguayante (partículas) y Cabrero (MDF) respectivamente. 


\section{CUADRO 1}

Parámetros del proceso para la fabricación de tableros de partículas con eucalipto nitens. Condiciones fijadas como para la producción con pino radiata.

Processing parameters for particleboard production using Eucalyptus nitens. Conditions fixed as for production using Pinus radiata.

\begin{tabular}{|c|c|c|c|}
\hline Sección & Item & Unidad & Valor \\
\hline Madera & $\begin{array}{l}\text { Flujo alimentación madera } \\
\text { Aserrín } \\
\text { Cantidad de partículas HOMBACK }\end{array}$ & $\begin{array}{c}\mathrm{kg} / \mathrm{min} \\
\% \\
\%\end{array}$ & $\begin{array}{r}101 \\
80 \\
20\end{array}$ \\
\hline Secado & $\begin{array}{l}\text { Temperatura cámara combustión } \\
\text { Temperatura entrada pre secador } \\
\text { Temperatura salida secador } \\
\text { Humedad partículas secas }\end{array}$ & $\begin{array}{l}{ }^{\circ} \mathrm{C} \\
{ }^{\circ} \mathrm{C} \\
{ }^{\circ} \mathrm{C} \\
\%\end{array}$ & $\begin{array}{r}850 \\
363 \\
150 \\
2,9\end{array}$ \\
\hline Encolado & $\begin{array}{l}\text { Razón de encolado capa media } \\
\text { Razón de encolado capa superficial } \\
\text { Catalizador capa media } \\
\text { Hidrófobo capa media } \\
\text { Hidrófobo capa superficial } \\
\text { Humedad capa media encolada } \\
\text { Humedad capa superficial encolada }\end{array}$ & $\begin{array}{l}\% \\
\% \\
\% \\
\% \\
\% \\
\% \\
\%\end{array}$ & $\begin{array}{r}7,2 \\
11,0 \\
0,9 \\
0,64 \\
0,32 \\
9,7 \\
16,2\end{array}$ \\
\hline Estado de la estera & $\begin{array}{l}\text { Densidad material capa superficial } \\
\text { Densidad material capa media } \\
\text { Finos en el tablero }\end{array}$ & $\begin{array}{c}\mathrm{kg} / \mathrm{m}^{3} \\
\mathrm{~kg} / \mathrm{m}^{3} \\
\%\end{array}$ & $\begin{array}{r}190 \\
160 \\
44,2\end{array}$ \\
\hline Prensado & $\begin{array}{l}\text { Presión en el preprensado } \\
\text { Tiempo de preprensado } \\
\text { Temperatura prensado } \\
\text { Presión máxima } \\
\text { Factor de prensado } \\
\text { Prensadas por turno } \\
\text { Peso tablero salida prensa }\end{array}$ & $\begin{array}{c}\text { bar } \\
\mathrm{s} \\
{ }^{\circ} \mathrm{C} \\
\mathrm{bar} \\
\mathrm{s} / \mathrm{mm} \\
\mathrm{N}^{\mathrm{o}} \\
\mathrm{kg}\end{array}$ & $\begin{array}{r}100 \\
5,5 \\
181 \\
290 \\
15 \\
100 \\
88,2\end{array}$ \\
\hline
\end{tabular}

\section{RESULTADOS}

Densidad de la madera: Antes de realizar las pruebas industriales se controló la densidad de la madera de E. nitens y se comparó con el promedio que la empresa ha determinado para su mezcla de $P$. radiata. Los resultados se presentan en el cuadro 3.

Los valores de densidad registrados son similares a los determinados por otros autores. En un proyecto desarrollado por INFOR (3) se determinaron densidades de $435 \mathrm{~kg} / \mathrm{m}^{3}$ y $495 \mathrm{~kg} / \mathrm{m}^{3}$ para E. nitens "juvenil" y "adulto" respectivamente. En ensayos para contrachapados, Poblete (4) in- forma de una densidad promedio de $470 \mathrm{~kg} / \mathrm{m}^{3}$ para árboles de 10 y 17 años.

Respecto a las densidades básicas determinadas en este estudio, la madera de E. nitens de 5 años poseía una densidad $8 \%$ mayor que la madera de pino normalmente utilizada por las plantas industriales. La madera de E. nitens de 13 años registró una densidad básica $12 \%$ mayor que la del P. Radiata.

Aun cuando las diferencias entre las densidades de $P$. Radiata y E. nitens son menores, este cambio en la densidad de la materia prima debería tener como consecuencia una corrección en los parámetros de producción. Al aumentar la den- 


\section{CUADRO 2}

Parámetros del proceso para la fabricación de tableros MDF con eucalipto nitens. Condiciones fijadas como para la producción con pino radiata. Processing parameters for MDF production. Conditions fixed as for production using Pinus radiata.

\begin{tabular}{|l|c|c|}
\hline Item & Unidad & Valor \\
\hline Humedad de las fibras en línea & $\%$ & 12 \\
Altura Scalper* & $\mathrm{mm}$ & 202 \\
Ancho master panel MDF & $\mathrm{mm}$ & 2.530 \\
Largo master panel MDF & $\mathrm{mm}$ & 1.482 \\
Peso master panel MDF & $\mathrm{kg}$ & 107,3 \\
Temperatura zona 1 & ${ }^{\circ} \mathrm{C}$ & 205 \\
Temperatura zona 2 & ${ }^{\circ} \mathrm{C}$ & 208 \\
Temperatura zona 3 & ${ }^{\circ} \mathrm{C}$ & 210 \\
Flujo de madera (base seca) & $\mathrm{Ton} / \mathrm{h}$ & 12,7 \\
Resina & $\%$ & 17 \\
Urea & $\%$ & 15 \\
Hidrófobo & $\%$ & 0,9 \\
\hline
\end{tabular}

*: Scalper: Rodillo a la salida de la formadora que determin la altura de la estera de fibras.

\section{CUADRO 3}

Densidad básica de la madera de Eucalyptus nitens y de Pinus radiata.

Specific weight of Eucalyptus nitens and Pinus radiata wood.

\begin{tabular}{|c|c|c|}
\hline Especie & Edad & Densidad $\left(\mathrm{kg} / \mathrm{m}^{3}\right)$ \\
\hline \multirow{2}{*}{ Eucalyptus nitens } & 5 & 430 \\
\cline { 2 - 3 } & 13 & 450 \\
\hline Pinus radiata & Se desconoce & 400 \\
\hline
\end{tabular}

sidad de la madera empleada y mantener constante la densidad del tablero producido, se provoca una disminución en la razón de compresión. Este efecto podría causar un descenso en algunas propiedades del producto y se corrige aumentando la densidad del tablero.

Producción y propiedades de tableros de partículas: Las observaciones que se realizaron durante el procesamiento de la madera permitieron comprobar que el flujo de producción, durante los cuatro turnos que duró el ensayo, no sufrió alteraciones.

En esta etapa de la producción el parámetro más importante a controlar era el tamaño de las partículas que se generaba con la nueva especie. Los resultados de este análisis se presentan en la figura 1 .

Los resultados presentados en la figura 1 permiten apreciar diferencias en la distribución de tamaños de las virutas obtenidas al cortar las trozas con la viruteadora. Se determinó que existe una mayor cantidad de virutas mayores a $5 \mathrm{~mm}$ en la madera de 13 años. Esta característica provocada por las edades de E. nitens no tiene una influencia significativa en las propiedades físico-mecánicas de los tableros. No es posible identificar la causa de esta diferencia entre los tamaños de partículas, las posibles causas podrían ser que el equipo está calibrado para procesar trozas de un diámetro significativamente menor al de E. nitens, que la densidad de esta madera es mayor a la de $P$. Radiata y/o que la humedad de las trozas haya sido diferente.

Al comparar los tamaños de las partículas de E. nitens con las de P. Radiata (cuadro 4), se pudo observar que la distribución de tamaños obtenida con E. nitens de 13 años es semejante a la del P. Radiata.

A medida que se fueron produciendo tableros con la incorporación de E. nitens, se fueron tomando muestras para un control en línea. Posteriormente los tableros se dejaron reposar y se efectuaron los ensayos de acuerdo con las normas EN mencionadas anteriormente.

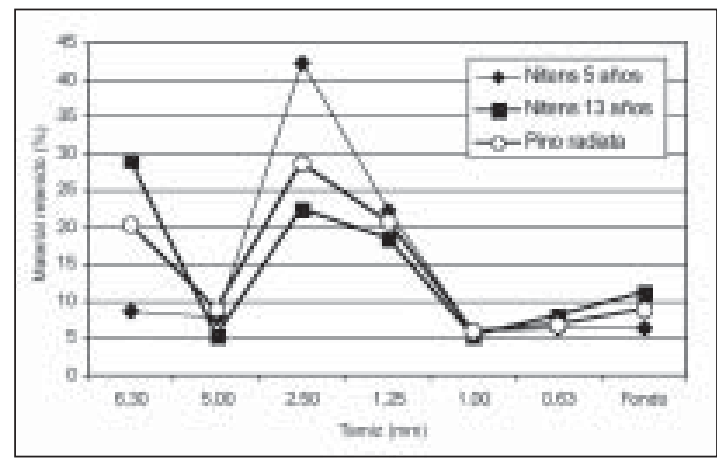

Figura 1. Distribución de tamaños de partículas obtenidas de metro ruma de Eucalyptus nitens y Pinus radiata.

Size distribution of particles produced from pulped logs of Eucalyptus nitens and Pinus radiata. 
BOSQUE 25(3): 89-97, 2004

Tableros de partículas y MDF de Eucalyptus nitens: Ensayos a escala industrial

Los resultados de los ensayos de las propiedades físicas y mecánicas se presentan en los cuadros 4 y 5 .

De los resultados obtenidos se puede apreciar que, en general, no existen diferencias significativas en las propiedades físico-mecánicas entre los tableros de 5 y de 13 años, fabricados con $E$. nitens.

En el cuadro 4 se aprecia que los tableros que contienen nitens alcanzaron densidades inferiores a la que se pretendía obtener $\left(640 \mathrm{~kg} / \mathrm{m}^{3}\right)$. La reducción de la densidad de los tableros con E. nitens

\section{CUADRO 4}

Propiedades físicas de tableros de partículas con $20 \%$ de Eucalyptus nitens y comparación con tableros con $100 \%$ Pinus radiata.

Physical properties of particleboard containing 20\% Eucalyptus nitens and comparison with 100\% Pinus radiata boards

\begin{tabular}{|c|c|c|c|c|c|c|c|c|}
\hline \multirow{3}{*}{ Tablero } & \multirow{3}{*}{ Parámetro } & \multirow[t]{2}{*}{ Densidad } & \multicolumn{2}{|c|}{ Hinchamiento } & \multicolumn{2}{|c|}{ Absorción } & \multirow[b]{2}{*}{ Humedad } & \multirow{2}{*}{$\begin{array}{l}\text { Emisión } \\
\text { HCHO }\end{array}$} \\
\hline & & & 2 horas & 24 horas & 2 horas & 24 horas & & \\
\hline & & $\mathrm{kg} / \mathrm{m}^{3}$ & $(\%)$ & $(\%)$ & $(\%)$ & $(\%)$ & $(\%)$ & $\mathrm{mg} / 100 \mathrm{~g}$ \\
\hline \multirow{3}{*}{ E. nitens 5 años } & Promedio & 633 & 6,7 & 19,2 & 27,6 & 69,3 & 8,8 & 8,9 \\
\hline & Desv. Estándar & 12 & 1,2 & 2,2 & 9,4 & 9,3 & 0,4 & 0,2 \\
\hline & Coef. Variación & 1,9 & 18,3 & 11,4 & 34,3 & 13,4 & 4,0 & 2,6 \\
\hline \multirow{3}{*}{ E. nitens 13 años } & Promedio & 636 & 5,1 & 17,6 & 21,8 & 60,6 & 9,2 & 8,5 \\
\hline & Desv. Estándar & 9,4 & 0,8 & 1,5 & 6,2 & 3,8 & 0,7 & 0,2 \\
\hline & Coef. Variación & 1,5 & 16,4 & 8,4 & 28,4 & 6,3 & 7,2 & 1,8 \\
\hline \multirow{3}{*}{$\begin{array}{l}\text { Producción normal } \\
\text { Pinus radiata }\end{array}$} & Promedio & 651 & 6,3 & 18,1 & 21,6 & 59,1 & 8,5 & 8,5 \\
\hline & Desv. Estándar & 6,6 & 1,2 & 1,9 & 4,3 & 4,5 & 0,5 & 0,3 \\
\hline & Coef. Variación & 1,0 & 22,4 & 10,5 & 19,7 & 7,6 & 6,3 & 3,5 \\
\hline Norma MASISA S.A. & & $640+/-20$ & $<8$ & $<20$ & $<25$ & $<80$ & $5-11$ & $<12$ \\
\hline
\end{tabular}

\section{CUADRO 5}

Propiedades mecánicas de tableros de partículas con $20 \%$ de Eucalyptus nitens y comparación con tableros con $100 \%$ Pinus radiata.

Mechanical properties of particleboard containing 20\% Eucalyptus nitens and comparison with 100\% Pinus radiata boards.

\begin{tabular}{|c|c|c|c|c|c|c|c|c|}
\hline \multirow{3}{*}{ Tablero } & \multirow{3}{*}{ Parámetro } & \multirow[b]{2}{*}{ Densidad } & \multicolumn{2}{|c|}{ Flexión estática } & \multirow[b]{2}{*}{ Tracción } & \multirow[b]{2}{*}{ Desp. Sup. } & \multicolumn{2}{|c|}{ Extracción tornillo } \\
\hline & & & MOR & MOE & & & cara & canto \\
\hline & & $\mathrm{kg} / \mathrm{m}^{3}$ & $\mathrm{~N} / \mathrm{mm}^{2}$ & $\mathrm{~N} / \mathrm{mm}^{2}$ & $\mathrm{~N} / \mathrm{mm}^{2}$ & $\mathrm{~N} / \mathrm{mm}^{2}$ & $\mathrm{~N}$ & $\mathrm{~N}$ \\
\hline \multirow{3}{*}{ E. nitens 5 años } & Promedio & 633 & 14,6 & 1.600 & 0,60 & 1,35 & 1.086 & 911 \\
\hline & Desv. Estándar & 12 & 0,7 & 170 & 0,07 & 0,14 & 142 & 62 \\
\hline & Coef. Variación & 1,9 & 4,7 & 10,6 & 12,2 & 10,7 & 13,1 & 6,9 \\
\hline \multirow{3}{*}{ E. nitens 13 años } & Promedio & 636 & 14,3 & 1.611 & 0,57 & 1,21 & 1.058 & 846 \\
\hline & Desv. Estándar & 9,4 & 1,2 & 36,7 & 0,07 & 0,13 & 97 & 65 \\
\hline & Coef. Variación & 1,5 & 8,4 & 2,3 & 12,7 & 10,9 & 9,1 & 7,7 \\
\hline \multirow{3}{*}{$\begin{array}{l}\text { Producción normal } \\
\text { Pinus radiata }\end{array}$} & Promedio & 651 & 16,5 & 1.785 & 0,61 & 1,46 & 1.248 & 891 \\
\hline & Desv. Estándar & 6,6 & 0,7 & 194,0 & 0,1 & 0,1 & 84,3 & 68,8 \\
\hline & Coef. Variación & 1,0 & 4,1 & 10,9 & 16,7 & 8,8 & 6,8 & 7,7 \\
\hline Norma MASISA S.A. & & $640+/-20$ & $>16$ & $>1.300$ & $>0,5$ & $>1$ & $>900$ & $>800$ \\
\hline
\end{tabular}


de 5 y 13 años es 18 y $15 \mathrm{~kg} / \mathrm{m}^{3}$ respectivamente. En el caso de los tableros de partículas, una reducción de la densidad normalmente se traduce en un descenso en las propiedades mecánicas y un aumento de la capacidad de absorción de agua (5). Este tipo de efecto se observa en los resultados presentados en el cuadro 4, donde la absorción de agua de los tableros con E. nitens de 5 años y menor densidad es mayor a la de los tableros con E. nitens de 13 años y a los de $P$. radiata. Por lo anterior se puede pronosticar que, al aumentar la densidad de los tableros con E. nitens, esta propiedad debería mejorar. Pese a lo anterior, los tableros que contienen madera de $E$. nitens cumplen con los estándares de MASISA S.A.

En el caso del hinchamiento (cuadro 4) la literatura indica que por lo general no existe una relación con la densidad del tablero. Esta propiedad queda definida por aptitudes propias de la especie, el tipo de adhesivo y los aditivos que se agreguen. En el caso del presente estudio no se observan diferencias de importancia y los tableros con incorporación de nitens cumplen con los requisitos de la empresa (ver última fila en cuadro 4).

La emisión de formaldehido de los tableros estudiados se presenta en el cuadro 4. La especie de madera que se emplee puede tener un efecto menor sobre esta propiedad, dependiendo del tipo de extraíbles que la compongan o de la cantidad de corteza que se agregue con la madera. En este caso la madera estaba descortezada y el contenido y tipo de extraíbles del eucalipto no juegan un papel de importancia. Esta propiedad se determinó con el objeto de cumplir con los controles normales de la planta. Todos los tableros cumplieron con las exigencias de la planta y de la norma EN.

Como se mencionó, la densidad del tablero tiene un efecto directo sobre las propiedades mecánicas. Por esta razón, los valores presentados en el cuadro 5 deberían mejorar al aumentar la densidad en los aproximadamente $20 \mathrm{~kg} / \mathrm{m}^{3}$ que faltan para cumplir con la densidad nominal.

Al comparar los resultados obtenidos por los tableros que contienen $E$. nitens con tableros fabricados con $100 \%$ de pino radiata (cuadro 5) se observa que la resistencia a la flexión se ve reducida en aproximadamente $2 \mathrm{~N} / \mathrm{mm}^{2}$. Considerando los parámetros de producción empleados (cuadro 1), normalmente por cada $10 \mathrm{~kg} / \mathrm{m}^{3} \mathrm{de}$ aumento en la densidad se provoca un aumento de $0,8 \mathrm{~N} / \mathrm{mm}^{2}$ en la flexión. Por lo tanto, los valores esperados de flexión con $E$. nitens, con la densidad nominal, serían de aproximadamente $16 \mathrm{~N} / \mathrm{mm}^{2}$ y cumplirían con la norma.

Los valores de tracción, desprendimiento superficial y de extracción de tornillos superan las exigencias y no se aprecian diferencias importantes entre ellos.

En términos generales, los tableros fabricados con un $20 \%$ de E. nitens cumplirían las normas de propiedades físico-mecánicas garantizadas por la empresa MASISA S.A. para sus tableros.

Cabe mencionar que, en cuanto al aspecto de las superficies, en los tableros que contienen nitens es posible apreciar la presencia de "agujas" en su superficie, lo que le da una terminación menos homogénea que la del tablero fabricado sólo con $P$. radiata.

Posterior a la fabricación de los tableros, un 90\% de la producción de los aglomerados fue recubierto con papel melamínico. Al evaluar, con los estándares de MASISA S.A., la calidad de estos recubrimientos se pudo constatar que ellos presentaban el mismo nivel de calidad que los tableros normales. Estos tableros recubiertos fueron comercializados al mismo precio de venta que la placa de melamina tradicional, sin inconvenientes para los clientes.

Producción y propiedades de tableros MDF: Como se mencionara, la producción de estos tableros se realizó con astillas de E. nitens reemplazando en un $100 \%$ a las de $P$. radiata. Pese a este importante cambio, y a haber mantenido todas las condiciones de fabricación que se aplican para radiata, no se detectaron interrupciones en el proceso.

En cuanto a la calidad de las fibras entregadas por E. nitens, se observó una distribución del tamaño de fibras diferente a la distribución que presenta el $P$. radiata (figura 2).

Se observa en la figura 2 que la distribución de fibras en el caso de $P$. radiata sigue la forma de una campana, mientras que en nitens se da una mayor proporción de las fibras de menor área para luego disminuir a medida que crece el tamaño del área. Al final del gráfico, nitens presenta una mayor cantidad de material grueso (haces de fibras o shives) que radiata. 


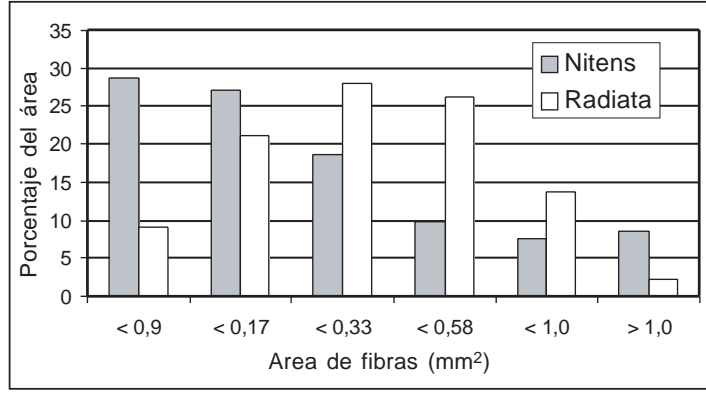

Figura 2. Area de las fibras (termomecánica: Sund Defibrator) del material producido con Eucalyptus nitens y Pinus radiata.

Fiber area (thermomechanical: Sunds Defibrator) of material produced using Eucalyptus nitens and Pinus radiata.

En general, la distribución de tamaños de fibras de $E$. nitens es significativamente diferente a la de pino radiata. Tratándose E. nitens de una latifoliada, era esperable obtener tamaños menores, pero con una distribución normal (campana de Gauss). Esta irregularidad se debe, sin duda, a que tras años de experiencia, las condiciones del desfibrado y hasta el diseño de los discos del molino están ajustados para procesar una conífera: el $P$. radiata. En otras palabras, las condiciones de desfibrado no son las óptimas para $E$. nitens; para trabajar con esta especie se deben mejorar las condiciones del tratamiento hidrotérmico y de la etapa mecánica del desfibrado. Hechas estas correcciones se mejorará tanto el tipo de fibras obtenidas como también las propiedades del producto.

La mayor cantidad de haces de fibras, shives, no es deseable, ya que influyen negativamente en la calidad de la superficie del tablero como también en su respuesta al fresado. Por otra parte, la mayor cantidad de finos producidos en el proceso de desfibrado puede influir desfavorablemente en el consumo de adhesivo, o bien, provocando una disminución de las propiedades físicas y mecánicas.

Los resultados de las mediciones de propiedades físicas y mecánicas se presentan en los cuadros 6 y 7 , y muestran que prácticamente no existe influencia de la edad de la madera de E. nitens. Esto es una ventaja para la madera de 5 años, ya que se obtienen las mismas propiedades que con un material cosechado 8 años más tarde.

En general, al comparar las propiedades de los tableros de nitens con los fabricados con astillas de $P$. radiata, se observa que los de $E$. nitens son inferiores en prácticamente todas las propiedades.

En el cuadro 6 se presentan los resultados de las propiedades físicas. Se observa que la densidad promedio de los tableros de E. nitens de 5 años es inferior, por lo que al corregir esta diferencia se deberían mejorar sus propiedades. Si se

\section{CUADRO 6}

Propiedades físicas de tableros MDF de Eucalyptus nitens y comparación con tableros de Pinus radiata. Physical properties of Eucalyptus nitens MDF boards and comparison with Pinus radiata boards.

\begin{tabular}{|c|c|c|c|c|c|c|c|c|}
\hline \multirow[t]{2}{*}{ Tablero } & \multirow[t]{2}{*}{ Parámetro } & Densidad & Velocidad & $\begin{array}{l}\text { Humedad } \\
\text { Línea }\end{array}$ & $\begin{array}{c}\text { Espesor } \\
\text { Bruto }\end{array}$ & $\begin{array}{c}\text { Hinchamiento } \\
24 \text { horas }\end{array}$ & $\begin{array}{l}\text { Absorción } \\
24 \text { horas }\end{array}$ & Humedad \\
\hline & & $\mathrm{kg} / \mathrm{m}^{3}$ & $\mathrm{~m} / \mathrm{min}$ & $\%$ & $\mathrm{~mm}$ & $\%$ & $\%$ & $\%$ \\
\hline \multirow{3}{*}{ E. nitens 5 años } & Promedio & 611 & 7,6 & 11,2 & 16,2 & 7,1 & 26,6 & 7,1 \\
\hline & Desv. Estándar & 11,22 & 0,50 & 0,30 & 0,25 & 0,63 & 3,52 & 0,75 \\
\hline & Coef. Variación & 1,8 & 6,5 & 2,7 & 1,5 & 8,8 & 13,2 & 10,6 \\
\hline \multirow{3}{*}{ E. nitens 13 años } & Promedio & 623 & 7,5 & 11,2 & 16,5 & 7,3 & 26,4 & 7,6 \\
\hline & Desv. Estándar & 10,1 & 0,25 & 0,10 & 0,19 & 0,6 & 1,1 & 0,95 \\
\hline & Coef. Variación & 1,6 & 3,3 & 0,9 & 1,2 & 7,8 & 4,2 & 12,5 \\
\hline \multirow{3}{*}{$\begin{array}{l}\text { Producción normal } \\
\text { Pinus radiata }\end{array}$} & Promedio & 624 & 10,5 & 12,3 & 16,1 & 5,8 & 15,0 & 6,7 \\
\hline & Desv. Estándar & 11,4 & 0,0 & 0,37 & 0,11 & 0,5 & 1,4 & 0,34 \\
\hline & Coef. Variación & 1,8 & 0,0 & 3,0 & 0,7 & 7,9 & 9,4 & 5,0 \\
\hline Norma MASISA S.A. & & $620+/-25$ & & & & $<10$ & $<22$ & $5-11$ \\
\hline
\end{tabular}


BOSQUE 25(3): 89-97, 2004

Tableros de partículas y MDF de Eucalyptus nitens: Ensayos a escala industrial

\section{CUADRO 7}

Propiedades mecánicas de tableros MDF de Eucalyptus nitens y comparación con tableros de Pinus radiata. Mechanical properties of Eucalyptus nitens MDF boards and comparison with Pinus radiata boards.

\begin{tabular}{|c|c|c|c|c|c|c|c|c|}
\hline \multirow{3}{*}{ Tablero } & \multirow{3}{*}{ Parámetro } & \multirow[t]{2}{*}{ Densidad } & \multicolumn{2}{|c|}{ Flexión estática } & \multicolumn{2}{|c|}{ Tracción } & \multicolumn{2}{|c|}{ Tornillo } \\
\hline & & & MOR & MOE & Prom. & Mínima & cara & canto \\
\hline & & $\mathrm{kg} / \mathrm{m}^{3}$ & $\mathrm{~N} / \mathrm{mm}^{2}$ & $\mathrm{~N} / \mathrm{mm}^{2}$ & $\mathrm{~N} / \mathrm{mm}^{2}$ & $\mathrm{~N} / \mathrm{mm}^{2}$ & $\mathrm{~N}$ & $\mathrm{~N}$ \\
\hline \multirow{3}{*}{ E. nitens 5 años } & Promedio & 611 & 26 & 2.093 & 0,68 & 0,6 & 869 & 776 \\
\hline & Desv. Estándar & 11,22 & 1,41 & 72 & 0,08 & 0,07 & 52 & 78 \\
\hline & Coef. Variación & 1,8 & 5,4 & 3,5 & 12,4 & 11,4 & 6 & 10,0 \\
\hline \multirow{3}{*}{ E. nitens 13 años } & Promedio & 623 & 27,5 & 2.249 & 0,65 & 0,59 & 951 & 788 \\
\hline & Desv. Estándar & 10,1 & 2,1 & 171 & 0,04 & 0,03 & 72 & 107 \\
\hline & Coef. Variación & 1,6 & 7,6 & 7,6 & 6,9 & 5,3 & 7,5 & 13,6 \\
\hline \multirow{3}{*}{$\begin{array}{l}\text { Producción normal } \\
\text { Pinus radiata }\end{array}$} & Promedio & 624 & 31,1 & 2.350 & 0,84 & 0,77 & 1.070 & 815 \\
\hline & Desv. Estándar & 11,4 & 4,9 & 302 & 0,10 & 0,11 & 116 & 88 \\
\hline & Coef. Variación & 1,8 & 15,7 & 12,8 & 12,0 & 14,9 & 10,9 & 10,8 \\
\hline Norma MASISA S.A. & & $620+1-25$ & $\min .30$ & $>2.000$ & $0,75+/-0,15$ & 0,52 & $>1.000$ & $>800$ \\
\hline
\end{tabular}

tiene presente que la densidad de la madera de $E$. nitens es mayor que la de $P$. radiata, en futuros ensayos debería considerarse la producción de tableros con una densidad mayor, para mantener o aumentar la razón de compresión y mejorar las propiedades.

Durante el proceso de fabricación se fueron tomando muestras para determinar propiedades on line y de esa forma se trató de corregir el proceso para asegurar el cumplimiento de los valores mínimos exigidos por la empresa. Entre las acciones que se decidió tomar estuvo reducir la velocidad de producción entre un 20 a un $25 \%$ (cuadro 6). Pese a lo anterior, no se logró obtener el promedio mínimo exigido para la absorción de agua (cuadro 6) y no se cumplieron las propiedades mecánicas flexión y extracción de tornillos (cuadro 7). Por la duración de los ensayos y la disponibilidad de madera no fue posible realizar otros cambios en el proceso que permitieran mejorar estas propiedades.

Los valores de hinchamiento (cuadro 6) son algo mayores que los que se obtienen con $P$. radiata, pero permiten cumplir con los estándares impuestos.

En el caso de la flexión y de la extracción de tornillos, es necesario tener presente que al incluir una proporción importante de fibras de ta- maño menor se provoca una mala distribución del adhesivo, vale decir, se tiene una mayor superficie específica para una cantidad constante de adhesivo. Esto demuestra que, aparte de la corrección requerida en la densidad del tablero, deben cambiarse las condiciones de desfibrado.

Al determinar los perfiles de densidad y comparar tableros con E. nitens y tableros con $P$. radiata, se observó que la densidad superficial del tablero con E. nitens fue del orden de 830 a $850 \mathrm{~kg} / \mathrm{m}^{3}$ y la densidad en el centro equivalía a un $89 \%$ respecto a la densidad media del tablero. En el caso del MDF con $P$. radiata la densidad superficial alcanzada era de 875 a $920 \mathrm{~kg} / \mathrm{m}^{3}$ y la densidad en el centro del tablero llegó a un $91 \%$ de la densidad media del tablero. Esto puede ser el resultado de tener una menor cantidad de adhesivo disponible, lo que impide una buena compactación. Además, la madera de nitens tiene una mayor densidad, por lo que se obtiene una menor compactación. Ambos efectos se corrigen modificando el desfibrado y elevando la densidad final del tablero. Otras posibilidades de corregir el proceso podrían ser otorgar condiciones para homogeneizar el perfil de densidad, disminuyendo la velocidad de cierre de la prensa y aumentando el contenido de humedad promedio de la estera. 
BOSQUE 25(3): 89-97, 2004

Tableros de partículas y MDF de Eucalyptus nitens: Ensayos a escala industrial

\section{CONCLUSIONES}

La densidad básica de la madera de Eucalyptus nitens de 5 años fue $8 \%$ mayor que la madera de $P$. radiata y la madera de $E$. nitens de 13 años tuvo una densidad básica $12 \%$ mayor que la del pino. Estas diferencias afectan el proceso de producción y la calidad de los tableros.

No existen diferencias significativas en las propiedades físico-mecánicas de los tableros fabricados con Eucalyptus nitens de 5 y de 13 años.

Todos los tableros de E. nitens registraron densidades promedio menores a las de los de radiata. En futuros ensayos se debe considerar aumentar la densidad con lo que se espera obtener aumentos en las propiedades.

Al comparar nitens con $P$. radiata se observó que hay diferencias en la distribución de tamaños de las partículas y fibras producidas. Esto demuestra que al trabajar con E. nitens deben corregirse el corte y desfibrado.

Los tableros de partículas fabricados con un $20 \%$ de Eucalyptus nitens cumplen las normas de propiedades físico-mecánicas garantizadas por MASISA S.A. para sus tableros. Los tableros MDF no cumplen con las especificaciones por lo que se debe adecuar el proceso de producción.

Los tableros con E. nitens presentan superficies que muestran agujas (tableros de partículas) o shives (MDF). Esto debe ser corregido al adecuar los parámetros de corte y desfibrado de la madera.

El perfil de densidad de los tableros MDF fabricados con E. nitens es menos homogéneo que el de los de $P$. radiata y debe ser mejorado.

\section{BIBLIOGRAFIA}

(1) ROZAS, C., R. SANCHEZ, C. MIRANDA. 2001. Resultados preliminares del secado de Eucaliptus nitens de 11 años de edad. En: Simposio Internacional IUFRO. Desarrollando el Eucalipto del Futuro. Valdivia, Chile, septiembre 2001, 95 p.

(2) POBLETE, H. 2002a. Estudio técnico de la utilización de Eucaliptus nitens en la fabricación de contrachapados: ensayo con trozas basales y segundas. Informe de Convenio. Universidad Austral de Chile-Forestal Mininco S.A., 42 p.

(3) INFOR. 1998. Secado y trabajabilidad de tres especies de eucaliptus crecidos en Chile. Módulo 4: Tableros enlistonados. Fondo Nacional de Desarrollo Tecnológico y Productivo. Informe Final Proyecto de Innovación Tecnológica. Código FONTEC: 96-0705, 9 p.

(4) POBLETE, H. 2002b. Estudio técnico de la utilización de Eucaliptus nitens en la fabricación de contrachapados: Segundo ensayo: árboles de 10 y 17 años. Informe de Convenio, Universidad Austral de Chile-Forestal Mininco S.A., $35 \mathrm{p}$.

(5) KELLY, M. 1977. Critical literature review of relationships between processing parameters and physical properties of particleboard. For. Prod. Lab. US Forest Service. FPL-10. 64 p. 